\title{
Relationship of personality characteristics and eating attitude with the success of bariatric surgery
}

\author{
Ahmad Tayefi ${ }^{1}$, Abdolreza Pazouki $^{1}$, Kaveh Alavi $^{2}$, Razieh Salehian ${ }^{1}$, Fahimeh Soheilipour ${ }^{1}$, Atefeh Ghanbari Jolfaei*1(D) \\ Received: 28 Jul 2019 \\ Published: 31 Jul 2020
}

\section{Abstract}

Background: Obesity is a chronic disease that causes several medical and psychiatric complications. There are plenty of pharmacological and non-pharmacological therapies for obesity treatment. Bariatric surgery is one of the most efficient nonpharmacological treatment for morbid obesity; however, several psychological factors affect the success of bariatric surgery. This study aims to evaluate personality characteristics and eating attitude relationship with the success of bariatric surgery.

Methods: This study was carried out on 75 patients with obesity who were candidates of bariatric surgery in the obesity clinic of Rasoul-e-Akram Hospital in Tehran. The patients were asked to fill the TCI and EAT-26 questionnaires before and after the surgery. Statistical analyses were performed using the SPSS-23 applying T-test, Mann-Whitney tests and Pearson and Spearman's correlation coefficient. The significance level was set at 0.05 .

Results: Seventy-five patients, including 13 men and 62 women, were assessed through this study. The mean of the BMI of the participants was 44.7 prior to the surgery and 30 after the operation. Personality characteristics and eating attitudes had no significant relationship with the success of bariatric surgery.

Conclusion: Although the eating attitude and personal characteristics of the bariatric surgery candidates before the surgery was not related to the outcome of the surgery, they should be considered in post-operational psychological assessments.

Keywords: Temperament, Eating attitude, Personality characteristics, Success, Bariatric surgery, Obesity

Conflicts of Interest: None declared

Funding: This project was supported financially by Minimally Invasive Surgery Research Center in Iran University of Medical sciences.

\section{*This work has been published under CC BY-NC-SA 1.0 license.}

Copyright $\odot$ Iran University of Medical Sciences

Cite this article as: Tayefi A, Pazouki A, Alavi K, Salehian R, Soheilipour F, Ghanbari Jolfaei A. Relationship of personality characteristics and eating attitude with the success of bariatric surgery. Med J Islam Repub Iran. 2020 (31 Jul);34:89. https://doi.org/10.47176/mjiri.34.89

\section{Introduction}

Obesity is a chronic disease recognized with fat accumulation in the body. Typically, obesity is determined by means of BMI (Body Mass Index) (1). According to the AHA (American Heart Association) classification, obesity is defined as BMI higher than 30 (2). Although the com-

Corresponding author: Dr Atefeh Ghanbari Jolfaei, ghanbari.a@iums.ac.ir

1. Minimally Invasive Surgery Research Center, Iran University of Medical Sciences, Tehran, Iran

2. Mental Health research center, Iran University of Medical Sciences, Tehran, Iran plications of obesity are generally physical, the psychological consequences such as lacking self-confidence, depression, anxiety, and degradation of the social and economic status are also prevalent.

In addition, obesity has a high rate of comorbidity with

$\uparrow$ What is "already known" in this topic:

There seems to be a correlation between personality traits and eating attitude with the outcome of obesity surgery, although different studies have shown contradictory results.

\section{$\rightarrow$ What this article adds:}

The results showed that there was no relationship between personality traits, food attitude, and success of bariatric surgery. It seems that revision of psychiatric evaluations before bariatric surgery is needed and adopting a supportive approach instead of eliminating patients with psychiatric problems and focusing more on providing psychological services after surgery is suggested. 
some psychiatric disorders such as eating disorders, substance use disorders, anxiety disorders, mood disorders, personality disorders, attention deficit and hyperactivity disorder, and post-traumatic stress disorder (1).

Obesity increases medical costs for both the individual and the community. For example, the cost of an obese person pays for his medical problems is $42 \%$ higher than the normal weight person. The high BMI (BMI> 30) accounts for $9.1 \%$ of the cost of health services in the United States and is estimated to reach $16 \%$ by $2030(1)$. Regarding the individual and community costs of obesity, one of the health priorities is the management of this chronic disease.

There are various invasive and non-invasive treatments for obesity. Bariatric surgery is indicated for the patients with BMI equals or higher than 40, who have failed in diet therapy or for those with BMI equals to 35 to 40 , who have some special medical comorbidities. While bariatric surgery can improve some of the obesity-related complications such as diabetes, hypertension, sleep apnea and arthritis, the risk of surgical failure is still considerable; for example, $15 \%$ of the individuals achieved significant weight gain 3 years after gastric bypass surgery because of an improper diet (2).

Given the risk and cost of surgery, it is very important to increase the chance of surgery success by considering its related factors. Some studies have shown a possible relationship between the outcome of bariatric surgery and psychological factors such as personality characteristics and eating habits (3-6).

Personality refers the characteristics that change the patterns of thinking, feeling and behaving. Temperament is the hereditary part, and character is the acquired part of the personality (7). There seems to be a correlation between personality traits and the outcome of obesity surgery, although different studies have shown contradictory results $(8,9)$.

Two studies carried out by Chiara De Panfilis et al. and Gordon et al have shown a significant correlation between the perseverance and the BMI reduction after obesity surgery; however, no significant correlation between personality characteristics and outcome of bariatric surgery was found in some other studies $(3,5,10,11)$.

The other important factors that are assumed to affect the outcome of the obesity surgery are eating habits and attitude. Some studies have described that the anatomical changes after surgery can help to correct eating habits by setting some physical limitations. Pre-operative eating habits do not play a significant role in the outcome of surgery (12-14), while some other studies have shown opposite results (15).

The psychological assessment is included in most presurgery evaluation protocols; for example, in $90 \%$ of bariatric surgeries performed in the Netherland, a mental health specialist has been involved (16). The precise discernment of the psychological factors which are correlated with the outcome of bariatric surgery can be used as a guide to plan such assessments.

Previous studies have shown that eating disorders and eating habits are culturally dependent. Moreover, the cul- ture in which one grows up affects personality traits such as collectivism, individualism, complexity, tightness and openness (17).

According to the contrary results of the previous studies and regarding the impact of cultural issues on psychological factors, we conclude that more studies are needed, especially in different countries. The present study aimed to examine the possible relationship between the personality traits and eating attitude with the outcome of bariatric surgery.

\section{Methods}

This was a prospective, observational, follow up study. All of the patients were admitted to the outpatient obesity clinic of Rasoul-e-Akram hospital, a training hospital affiliated to Iran University of medical sciences.

The study was carried out on 93 candidates of bariatric surgery after approval by the ethical committee of Iran University of Medical Sciences, Tehran, Iran. All the participants were informed about the conditions of the study. Only candidates who expressed their informed consent and were older than 18 years old were included. Uncorrected hormone problems like adrenal or thyroid dysfunction and major psychiatric disorders were exclusion criteria. Roux-en-Y gastric bypass was performed by one surgeon (Abdolreza Pazouki, M.D.) at Rasoul-e-Akram hospital.

Psychiatric evaluation of all patients was done through the Structured Clinical Interview for DSM-IV Axis I Disorders (SCID-I) by a psychiatrist who was a faculty member of Iran University of Medical Sciences.

A week before surgery, the BMI of the patients was calculated. In addition, the personality of the patients was assessed by the Temperament /Character Inventory (TCI), moreover Eating Attitudes Test (EAT-26) was employed to evaluate eating attitude. One year after the operation, a follow-up visit was set up with each patient.

The post-operative evaluation included the outcome of the surgery, assessed by Bariatric Analysis and Reporting Outcome System (BAROS) and the eating attitude evaluated by the EAT 26.

Statistical analyses were performed using the SPSS23. According to the distribution of data by using the data distribution chart and the Kolmogorov-Smirnov test, the appropriate test was selected. T-student, Mann-Whitney tests and Pearson and Spearman's correlation coefficient were applied to evaluate relationships between variables. To determine the significance of the differences, the alpha error was considered to be 0.05 .

\section{Measures}

Temperament and Character Inventory (TCI): TCI was developed by Robert Cloninger and was designed to determine personality temperaments and characters. This questionnaire evaulates seven dimensions of personality traits (including harm avoidance, novelty seeking, reward dependence, persistence, cooperativeness, selfdirectedness, and self-transcendence) that are implemented through 125 questions.

The validity and reliability of the Persian version of this 
questionnaire was reported by Kaviani et al. The Cronbach's alpha for each dimension was demonstrated as follows: $\mathrm{NS}=0.86, \mathrm{HA}=0.88, \mathrm{RD}=0.73, \mathrm{P}=0.79, \mathrm{CO}=0.86$, $\mathrm{SD}=0.86$, and $\mathrm{ST}=0.86$. Also the validity coefficients were reported as follows: $\mathrm{NS}=0.75, \mathrm{HA}=0.72, \mathrm{RD}=0.87$, $\mathrm{p}=0.9, \mathrm{CO}=0.76, \mathrm{SD}=0.66$, and $\mathrm{ST}=0.86(18)$.

Eating Attitudes Test (EAT-26): The primary version of the Eating Attitudes Test was provided by Garner et al. in 1979 with 40 phrases. The authors decided to shorten the items to 26 in 1982. The recent edition has been applied through many studies and has been a useful screening tool with high validity and reliability to assess eating disorder in different populations.

EAT-26 included 3 subscales: dieting, bulimia and food preoccupation, and oral control. The cutoff point of 20 or more is addressed as the high risk for the eating disorders (19).

Previous studies demonstrated validity and reliability of the Persian version of the questionnaire (20).

Bariatric Analysis and Reporting Outcome System (BAROS): This questionnaire is used for assessing the outcome of bariatric surgery. It defines five outcome groups (failure, fair, good, very good, and excellent) based on the scoring of three main subscales, including percentage of excess weight loss, changes in medical conditions, and quality of life $(21,22)$.

The Persian Structured Clinical Interview for DSM-IV axis I disorders (SCID-I): It is a diagnostic instrument which should be administered by a clinician. The Persian version was shown to have acceptable reliability, validity and feasibility on a large sample of Iranian patients; it was used to exclude the patients with major psychiatric comorbidities (23).

\section{Results}

From the 93 patients, only 75 patients completed the assessment one year after the surgery. Five patients decided to not undertake the operation and 13 did not attend follow up visit due to various reasons. The final sample included 13 males $(17.3 \%)$ and 62 females $(82.7 \%)$ with a mean age of $39.2 \pm 11.9$ years. The median age was 37 and the range was between 18 and 72 years old. $26.7 \%$ of the participants were single and $70.7 \%$ were married and the rest $(2.6 \%)$ were widowed or divorced. Among the partic- ipants, $21.3 \%$ were smokers.

The average BMIs of the studied patients before and one year after the surgery were $44.7 \pm 7.2 \mathrm{~kg} / \mathrm{m}^{2}$ and $30 \pm 6.6 \mathrm{~kg} / \mathrm{m}^{2}$, respectively. Pearson correlation coefficient between pre-operative BMI and post-operative BMI was $-0.734(p<0.001)$ and between pre-operative BMI and percentage of weight loss was $-0.533(p<0.001)$.

In terms of the outcome of bariatric surgery, the mean of total score of Baros, one year after surgery, was 4.2 \pm 2.2 (ranged from 2.4 to 8.7). According to the Baros's score, 8 patients $(10.7 \%)$ had surgical failure.

Regarding the changes in medical conditions after the surgery, 3 patients (4\%) reported exacerbation of their previous diseases, and $21(28.2 \%)$ did not report any changes in their medical condition. Besides, 30 patients $(40.0 \%)$ reported some improvement, 16 patients $(21.3 \%)$ reported recovery from at least one disease, and $5(6.7 \%)$ reported the recovery from all previous diseases.

The mean scores of different dimensions of personality traits were obtained using the Temperament and Character Inventory (TCI).

As shown in Table 1, there is no significant correlation between the score of the seven personality dimensions and Baros global score after surgery.

One year after surgery, the score of oral control and total score of the EAT-26 questionnaire were significantly higher than the pre-operative score $(p<0.001)$, but the scores of diet and bulimia items did not change significantly (p-values were 0.380 and 0.329 respectively).

As shown in Table 2, the pre-operative total score of of EAT-26 questionnaire and it's subscales did not show considerable correlation with Baros total score.

\section{Discussion}

The mean of BMI was 44.7 prior to the operation and 30 one year after the surgery. The reduction of BMI was statistically significant and demonstrated the efficacy of the operation on the BMI over a year.

Out of 16 smokers, only 5 kept on quitting smoking one year after surgery. Regarding the fact that smoking increases the risk of gastric complications such as dyspepsia and ulcers, it can be a considerable issue for clinicians to assess smoking in the follow up sessions.

The Pearson correlation coefficient between the pre-

Table 1. Correlation between temperament and character, and Baros global score

\begin{tabular}{lccc}
\hline Temperament/ Character & Mean \pm SD & Pearson coefficient (r) & P-value \\
\hline Harm Avoidance & $7.4 \pm 4.0$ & -0.106 & 0.367 \\
Novelty seeking & $8.3 \pm 3.1$ & -0.077 & 0.510 \\
Reward Dependence & $9.6 \pm 2.4$ & 0.055 & 0.642 \\
Persistence & $3.3 \pm 1.4$ & 0.076 & 0.519 \\
Self-directedness & $14.9 \pm 5.3$ & -0.025 & 0.832 \\
Cooperativeness & $18.6 \pm 3.0$ & -0.034 & 0.774 \\
Self-transcendence & $10.6 \pm 2.9$ & 0.119 & 0.308 \\
\hline
\end{tabular}

SD, standard deviation

Table 2. Pearson/Spearman correlation coefficients between the pre-operative score of EAT-26 and Baros total score

\begin{tabular}{lccc}
\hline Subscales of EAT-26 & Mean \pm SD & Correlation coefficient (r) & P-value \\
\hline Dieting & $11.8 \pm 6.1$ & 0.112 & 0.337 \\
Bulimia and food preoccupation & $1.5 \pm 2.1$ & 0.151 & 0.196 \\
Oral control & $2.9 \pm 2.9$ & -0.145 & 0.214 \\
Total score & $16.2 \pm 9.2$ & 0.085 & 0.470 \\
\hline
\end{tabular}


operation BMI and the weight loss ratio was -0.553 that shows that lower BMI scores will result in more weight loss ratio, which have consistency with the previous studies $(24,25)$.

Regarding the EAT scores, the mean of dieting subscale was 11.8 before and 10.9 one year after the surgery which was not statistically significant. It is interesting that despite notable weight loss, preoccupation about dieting was not changed, perhaps because of weight regain fear.

In spite of our expectation, the Bulimia's score after the surgery does not show remarkable decrease. One of the main phrases of Bulimia score in EAT-26 is "after meal vomiting", which may be considered as a purging method. Since nausea and vomiting may be common following bariatric surgery, the lack of significant change in Bulimia score would be understandable.

The average score of oral control increased from 2.9 to 8 ; it shows that bariatric surgery improves the control over eating and reduces the meal volume. Some phrases in oral control subscale about the recent weight loss may also count as the causes for score growth of oral control. In contrast to our study, in Guerdjikova et al. study, investigating mechanisms that are supposed to help overcoming emotional eating, was not changed 6 months after surgery (26).

The average total score of the eating attitude questionnaire was increased significantly after the operation, which is reasonable according to the increased score of oral control subscale.

Unexpectedly, the scores of eating attitude before surgery did not have significant correlation with the postoperation outcome which complies with some studies, but is incompatible with results of some other studies. For example, the results of the study of Colles et al. conducted on 129 patients using QEWP-R (Questionnaire on Eating and Weight Patterns-Revised), is in contrary to our study; they reported that pre-operative grazing has significant negative correlation with the post-operative weight loss ratio (15). That contradiction can be explained by the different questionnaires used for assessing the eating habits. For example, Colles et al. only applied BMI for the evaluation of surgery outcome, while in the present study Baros was exploited which excludes weight changes but includes other domains such as medical conditions and quality of life.

Although some studies suggest that comorbidity of eating disorders should be considered as a contraindication for the bariatric surgeries, some others such as Dymek et al. study reported that there isn't any significant correlation between pre-operative grazing and weight loss ratio 6 months after the surgery (12). Likewise, in Powers et al. study which has been carried out on 116 patients, 18 months after the operation no significant correlation between pre-operative eating disorders and weight loss was observed (13). These studies suggest that the surgery itself may reduce the pathological eating habits but the operation outcome does not link to the pre-operational eating disorders. Since we measured the eating habits and not eating disorders, one of the causes that we have not found any correlation between the surgery's success and eating habits may be related to the assessment method.

According to the results of current study, personality traits did not have any significant correlation with the success of the surgery. Incompatible with the current study, in Aguera et al. research carried out on 139 patients using TCI, the patients with high scores in cooperation scale lost more weight 2 years after the surgery (27). Additionally, studies performed by Panfilis et al. and Gordon et al. respectively carried out on 49 and 333 patients by TCI, have shown that greater score in persistency is correlated with more weight loss after the surgery $(3,5)$. Furthermore Shiri et al (28) reported that bariatric surgery could improve personality aspects such as sense of personal strength and interpersonal relationships.

In contrast with the above mentioned works, in the study done by Van Hout et al. which was carried out on 112 candidates of bariatric surgery using the Dependent Personality Questionnaire (DPQ), no significant correlation among the subscales of personality, weight loss, and development of quality of life was detected (11). Besides, in Canetti et al. study performed on 44 patients over one year using the NEO Personality Inventory, there was no significant correlation between the subscales of personality and post-operative weight regain (10). The diversity of conclusions could be rationalized as the following: Various studies, including the current study, have used different methods to evaluate the surgical outcomes and this may cause variation in the results

In addition, duration of the follow up in the current study was one year that is not long enough to evaluate the role of psychological factors. Some studies reported that pathological eating behaviors have improved after bariatric surgery, but they gotworse in long term significantly (29).

Furthermore, it seems that other factors such as surgeon's skill, surgical techniques and complications play important role in the surgical outcome.

Moreover, some variables not considered in the current study, like life style, exercising, compliance, family support and other medical, social and psychological variables can influence the results and the majority of our sample were female.

Planning more studies using psychiatric interview with longer follow up duration and more intervening variables is recommended.

\section{Conclusion}

Although the eating attitude and personal characteristics of the bariatric surgery candidates before the surgery does not predict outcome of the surgery, they should be considered in post-operational psychological assessments.

\section{Conflict of Interests}

The authors declare that they have no competing interests.

\section{References}

1. Sadock BJ, Sadock VA, Ruiz P. Synopsis of Psychiatry Behavioral Sciences Clinical Psychiatry. 11th ed. New York: Wolters Kluwer; 2015. $1058-77$ p. 
2. Charles Brunicardi F, Dana K, Timothy R, David L, John G, Jeffrey B, et al. schwartz's principles of surgery. 10th ed. United States of America: McGraw-Hill 2014. 1100-4 p.

3. Gordon PC, Sallet JA, Sallet PC. The impact of temperament and character inventory personality traits on long-term outcome of Rouxen-Y gastric bypass. Obes Surg. 2014;24(10):1647-55.

4. Pekkarinen T, Koskela K, Huikuri K, Mustajoki P. Long-term Results of Gastroplasty for Morbid Obesity: Binge-Eating as a Predictor of Poor Outcome. Obes Surg. 1994;4(3):248-55.

5. De Panfilis C, Cero S, Torre M, Salvatore P, Dall'Aglio E, Adorni A, et al. Utility of the temperament and character inventory (TCI) in outcome prediction of laparoscopic adjustable gastric banding: preliminary report. Obes Surg. 2006;16(7):842-7.

6. Generali I, De Panfilis C. Personality Traits and Weight Loss Surgery Outcome. Curr Obes Rep. 2018;7(3):227-34.

7. Sadock BJ, Sadock VA, Ruiz P. Synopsis of Psychiatry Behavioral Sciences Clinical Psychiatry. 11th ed. New York: Wolters Kluwer; 2015. 1485-95 p.

8. Ouellette AS, Rodrigue C, Lemieux S, Tchernof A, Biertho L, Begin C. An examination of the mechanisms and personality traits underlying food addiction among individuals with severe obesity awaiting bariatric surgery. Eat Weight Disord. 2017;22(4):633-40.

9. Marek RJ, Ben-Porath YS, Merrell J, Ashton K, Heinberg LJ. Predicting one and three month postoperative Somatic Concerns, Psychological Distress, and Maladaptive Eating Behaviors in bariatric surgery candidates with the Minnesota Multiphasic Personality Inventory-2 Restructured Form (MMPI-2-RF). Obes Surg. 2014;24(4):631-9.

10. Canetti L, Berry EM, Elizur Y. Psychosocial predictors of weight loss and psychological adjustment following bariatric surgery and a weight-loss program: the mediating role of emotional eating. Int J Eat Disord. 2009;42(2):109-17.

11. van Hout GC, Hagendoren CA, Verschure SK, van Heck GL. Psychosocial predictors of success after vertical banded gastroplasty. Obes Surg. 2009;19(6):701-7.

12. Dymek MP, le Grange D, Neven K, Alverdy J. Quality of life and psychosocial adjustment in patients after Roux-en-Y gastric bypass: a brief report. Obes Surg. 2001;11(1):32-9.

13. Powers PS, Perez A, Boyd F, Rosemurgy A. Eating pathology before and after bariatric surgery: a prospective study.Int $\mathrm{J}$ Eat Disord. 1999;25(3):293-300.

14. Matini D, Ghanbari Jolfaei A, Pazouki A, Pishgahroudsari M, Ehtesham M. The comparison of severity and prevalence of major depressive disorder, general anxiety disorder and eating disorders before and after bariatric surgery. Med $\mathrm{J}$ Islam Repub Iran. 2014;28(1):708-14.

15. Colles SL, Dixon JB, O'Brien PE. Grazing and loss of control related to eating: two high-risk factors following bariatric surgery. Obesity (Silver Spring, Md). 2008;16(3):615-22.

16. van Hout GC, Vreeswijk CM, van Heck GL. Bariatric surgery and bariatric psychology: evolution of the Dutch approach. Obes Surg. 2008;18(3):321-5.

17. Boutaud J, Becuţ A, Marinescu A. Food and culture. Cultural patterns and practices related to food in everyday life. Introduction. Int Rev Soc Res. 2016;6:1-3.

18. Kaviani H, Poor Naseh M. Validation Of Temperament And Character Inventory (TCI) In Iranian Sample: Normative Data. Tehran Univ Med J. 2005;63(2):89-98.

19. Pourghassem Gargari B, D K, Sajadi N S, Safoura K, Shahrokhi H. Risk of eating disorders in Tabrizian high school girls in 2007. Med J Tabriz Univ Med Sci Health Serv. 2009;30:21-6.

20. Parisa Aghagedi AE. Efficacy of instructing cognitive-behavioral stress management on attitude changes towards sub-scales of eating disorders among female students. Soc Cogn. 2014; Winter 2015:4458.

21. Favretti F, Cadiere GB, Segato G, Busetto L, Loffredo A, Vertruyen $\mathrm{M}$, et al. Bariatric analysis and reporting outcome system (BAROS) applied to laparoscopic gastric banding patients. Obes Surg. 1998;8(5):500-4.

22. Victorzon M, Tolonen P. Bariatric Analysis and Reporting Outcome System (BAROS) following laparoscopic adjustable gastric banding in Finland. Obes Surg. 2001;11(6):740-3.

23. Sharifi V, Assadi SM, Mohammadi MR, Amini H, Kaviani H, Semnani Y, et al. A Persian translation of the Structured Clinical Interview for Diagnostic and Statistical Manual of Mental Disorders,
Fourth Edition: psychometric properties. Compr Psychiatry. 2009;50(1):86-91.

24. Ma Y, Pagoto SL, Olendzki BC, Hafner AR, Perugini RA, Mason $\mathrm{R}$, et al. Predictors of weight status following laparoscopic gastric bypass. Obes Surg. 2006;16(9):1227-31.

25. Ochner CN, Jochner MC, Caruso EA, Teixeira J, Xavier Pi-Sunyer F. Effect of preoperative body mass index on weight loss after obesity surgery.Surg Obes Relat Dis. 2013;9(3):423-7.

26. Guerdjikova AI, West-Smith L, McElroy SL, Sonnanstine T, Stanford K, Keck PE, Jr. Emotional eating and emotional eating alternatives in subjects undergoing bariatric surgery. Obes Surg. 2007;17(8):1091-6.

27. Aguera Z, Garcia-Ruiz-de-Gordejuela A, Vilarrasa N, Sanchez I, Bano M, Camacho L, et al. Psychological and Personality Predictors of Weight Loss and Comorbid Metabolic Changes After Bariatric Surgery.Eur Eat Disord Rev. 2015;23(6):509-16.

28. Shiri S, Gurevich T, Feintuch U, Beglaibter N. Positive psychological impact of bariatric surgery. Obes Surg. 2007;17(5):663-8.

29. Nasirzadeh Y, Kantarovich K, Wnuk S, Okrainec A, Cassin SE, Hawa R, et al. Binge Eating, Loss of Control over Eating, Emotional Eating, and Night Eating After Bariatric Surgery: Results from the Toronto Bari-PSYCH Cohort Study. Obes Surg. 2018;28(7):2032-9. 